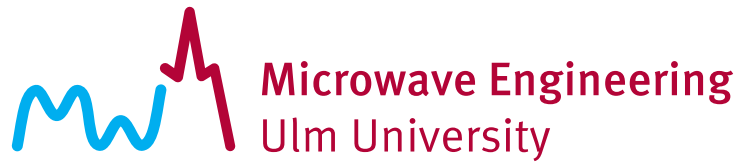

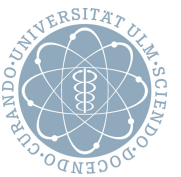

\section{Radar-Based Altitude over Ground Estimation of UAVs}

Markus Schartel, Ralf Burr, Pirmin Schoeder, Gilberto Rossi, Philipp Hügler, Winfried Mayer, and Christian Waldschmidt

(C) 2018 IEEE. Personal use of this material is permitted. Permission from IEEE must be obtained for all other uses, in any current or future media, including reprinting/republishing this material for advertising or promotional purposes, creating new collective works, for resale or redistribution to servers or lists, or reuse of any copyrighted component of this work in other works.

DOI: 10.23919/GEMIC.2018.8335039 


\title{
Radar-Based Altitude over Ground Estimation of UAVs
}

\author{
Markus Schartel*, Ralf Burr ${ }^{\dagger}$, Pirmin Schoeder*, Gilberto Rossi*, Philipp Hügler*, \\ Winfried Mayer ${ }^{\ddagger}$, and Christian Waldschmidt* \\ ${ }^{*}$ Institute of Microwave Engineering, Ulm University, 89081 Ulm, Germany \\ †Ulm University of Applied Sciences, 89075 Ulm, Germany \\ ‡Endress+Hauser GmbH+Co. KG, 79689 Maulburg, Germany \\ Email:markus.schartel@uni-ulm.de
}

\begin{abstract}
A $26 \mathrm{GHz}$ low-cost, low power, and low complexity pulse correlation radar (PCR) for online altitude over ground estimation of an unmanned aerial vehicle (UAV) is presented.

In the experimental part of this paper, measurement results of the customized lightweight radar are shown. Therefore, this radar, a commercially available lidar sensor, and a real time kinematic global navigation satellite system (RTK GNSS) are mounted on a UAV.

It is shown that in sparse vegetation, the radar in combination with a particle filter outperforms the lidar sensor. Besides, it is explained, why strong radar reflections can lead to a time offset of the particle filtered altitude. As an application, a radar-based terrain map is presented.
\end{abstract}

\section{INTRODUCTION}

In many applications the altitude above ground of a UAV has to be precisely known. By default, UAVs are equipped with pressure altimeters and GNSS systems. Both sensor principles provide an altitude information in relation to a reference plane. Pressure altimeters measure the changes in atmospheric pressure and use them to calculate the altitude. GNSS systems provide an altitude above a mathematical model that approximates the shape of the earth.

However, during the flight above rough terrain, e.g. upward and downward slopes, trenches, or undulating terrain, the altitude above ground, the so-called absolute altitude, cannot be measured by these sensors. Additionally, both sensor principles fail indoors, if there is no GNSS reception and the atmospheric pressure is influenced by air conditioning.

State-of-the-art consumer UAVs overcome this problem by using a downward looking vision system or ultrasonic sensors [1]. The drawback of these sensor principles is the limited measurement range, e.g. from $0.3 \mathrm{~m}$ to $13 \mathrm{~m}$ for the downward looking vision system, and the dependence on ambient conditions, e.g. temperature, light, and surface properties.

Many of these challenges can be overcome by using radar sensors. In [2] and [3] frequency modulated continuous wave (FMCW) radar altimeters are presented. However, FMCW radars are complex sensors. In order to record and process the output signal, a high-speed analog-to-digital converter (ADC) and a digital signal processor (DSP) to calculate the fast Fourier transform (FFT) are required.

In this paper a $26 \mathrm{GHz}$ low-cost radar sensor of low complexity for UAV online altitude estimation is presented. The advantage of this radar altimeter compared to an FMCW radar is the low power consumption, the low computational cost, and the low required ADC sampling frequency. The drawback is the comparatively low measurement rate.

The paper is organized as follows: The PCR is presented in Section II. Section III briefly describes the overall measurement system. In the Sections IV and V measurement results are presented and discussed. A radar-based terrain map is shown in Section VI. Section VII gives a short conclusion.

\section{Pulse Correlation Radar}

The block diagram of the $26 \mathrm{GHz}$ radar is shown in Fig. 1 . The radar front-ends are available as monolithically integrated radio frequency circuits. The functional principle of the PCR is explained in detail in [4]. The range to be observed is sequentially sampled by a varying time shift between the transmit oscillator (TX) and the local oscillator (LO) pulses.

The effective bandwidth of the radar is $1 \mathrm{GHz}$ resulting in a range resolution of about $15 \mathrm{~cm}$. The power consumption of this chip is $3 \mathrm{~mA}$, when supplied with a voltage of $3 \mathrm{~V}$. The pulse repetition frequencies $\left(f_{\mathrm{PRFS}}\right)$ determine the maximum unambiguous range $(80 \mathrm{~m})$ and the measurement rate $(10 \mathrm{~Hz})$. Instead of using two fine-tuned oscillators [5], the PRFs to trigger the transmit and the local oscillator are provided by a

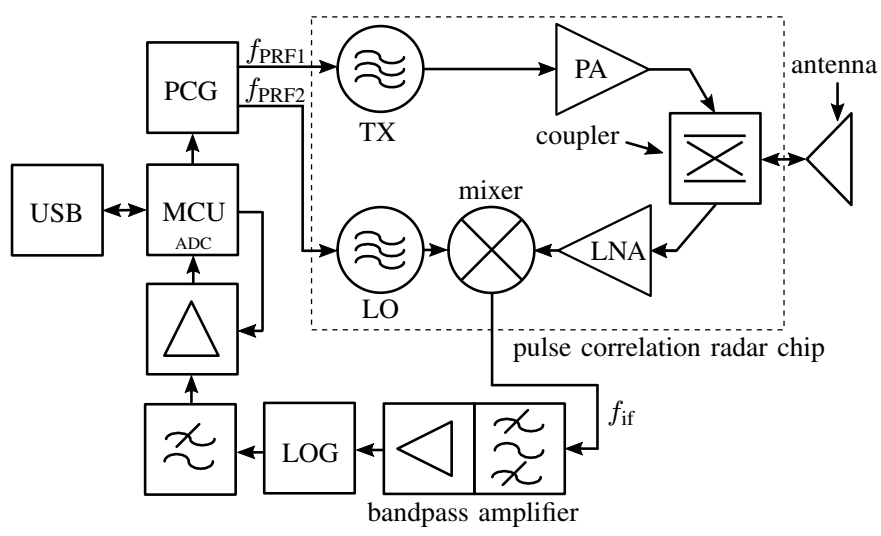

Fig. 1. Block diagram of the radar chip and its external circuit. The PCR consists of a transmit oscillator (TX), a local oscillator (LO), a power amplifier (PA), a coupler, a low noise amplifier (LNA), and a mixer. 
single programmable clock generator (PCG) chip. This allows the PRF to be changed dynamically during the flight. For instance, the measurement rate could be increased to $(40 \mathrm{~Hz})$ at the expense of the maximum unambiguous range $(20 \mathrm{~m})$ during take off and landing.

The intermediate frequency $\left(f_{\text {if }}\right)$ signal is amplified by a bandpass amplifier and a logarithmic detector (LOG). Subsequently, the signal is lowpass filtered with a cutoff frequency of $10 \mathrm{kHz}$, amplified, and sampled by a $20 \mathrm{kHz}$ microcontrollerintegrated ADC. The recorded raw radar signal, the so-called envelope curve (e.g. Fig. 8), can be interpreted without further signal processing. At the time of writing the envelope curve is simply forwarded via the universal serial bus (USB).

The size of the radar prototype excluding the antenna is about $53 \mathrm{~mm} \times 43 \mathrm{~mm} \times 10 \mathrm{~mm}$ and it weights $27 \mathrm{~g}$. The total power consumption of the radar sensor is about $480 \mathrm{~mW}$. For electromagnetic compatibility (EMC) reasons the radar is built into a tin shielding.

\section{Overall Measurement System}

The overall measurement system is shown in Fig. 2. As UAV a commercial available hexacopter is used. A differential RTK GNSS is mounted on top of the UAV and connected to the flight controller. The position data is broadcasted by the flight controller with an update rate of $5 \mathrm{~Hz}$ via the USB. The vertical positioning accuracy of this system is $2 \mathrm{~cm}$ [7].

The radar altimeter is mounted below the UAV. For measurement comparison, a lidar sensor is mounted next to it. The accuracy of the lidar is stated to be $\pm 2.5 \mathrm{~cm}$ up to $5 \mathrm{~m}$ distance and $\pm 10 \mathrm{~cm}$ beyond [6]. It runs with an update rate of $200 \mathrm{~Hz}$ and provides a single distance measurement value per measurement via inter-integrated circuit $\left(\mathrm{I}^{2} \mathrm{C}\right)$.

All measurements are recorded by a central data logger. It stores the RTK GNSS data, the lidar data, and the raw radar data and provides a time stamp to each measurement.

Since no gimbal is used, an inclination of the UAV results in a deviation of the measured altitude. Therefore, the sensors will illuminate an area that is not exactly below the UAV. This

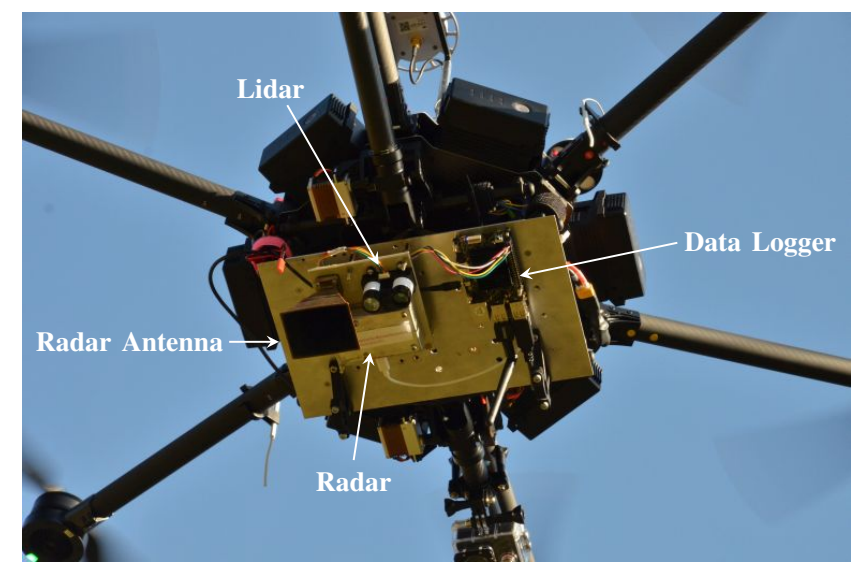

Fig. 2. DJI Matrice 600 Pro in conjunction with a DJI differential RTK GNSS. The radar altimeter, a lidar sensor, and a single-board computer are mounted below the UAV.

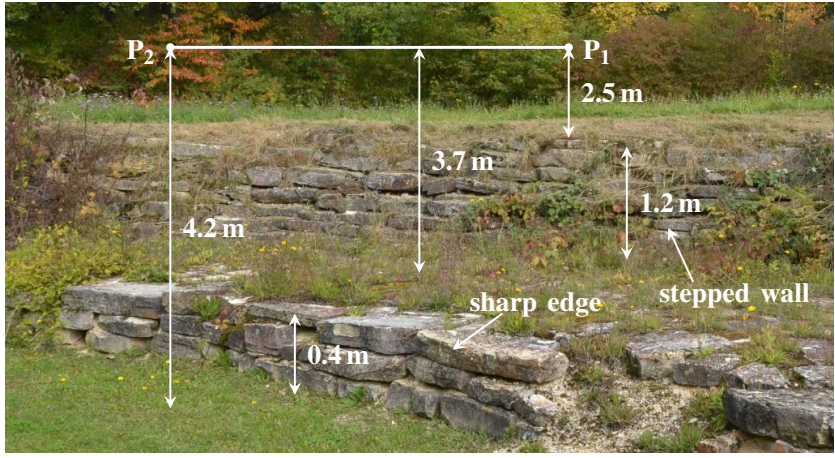

Fig. 3. Photo of the rock walls. The step size, the flight path, the different altitude levels, and the positions $P_{1}$ and $P_{2}$ are illustrated.

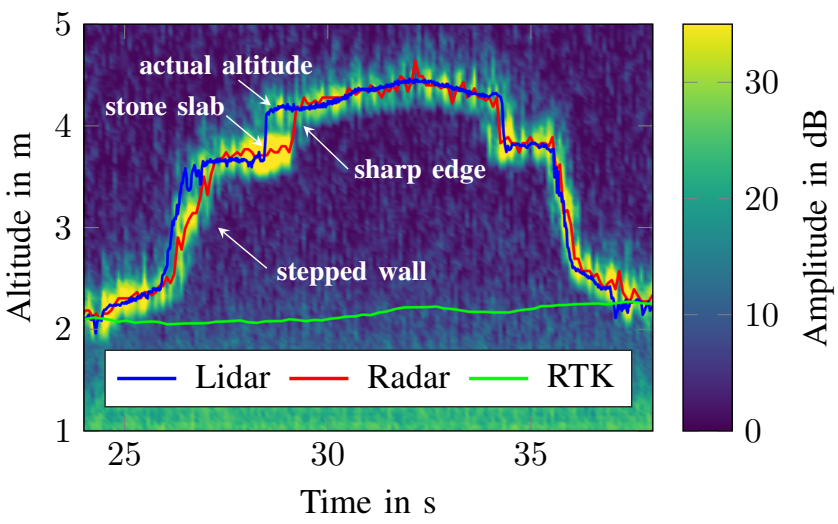

Fig. 4. Radar image from the flight above the rock walls.

error must be limited by a low airspeed, so that the UAV is as level as possible.

\section{EXPERIMENTAL RESULTS - ROCK WALL}

The UAV was flown horizontally over the two rock walls shown in Fig. 3. The first wall is $1.2 \mathrm{~m}$ high and is stepped, the second wall is $0.4 \mathrm{~m}$ high and has a sharp edge. From its take-off point, the UAV was steered to position $P_{1}$. From there it was flown above the stepped wall and the wall with a sharp edge to position $P_{2}$, and afterwards back to position $P_{1}$ again. The GNSS altitude of the UAV was kept constant at approximately $2 \mathrm{~m}$ above its take-off point.

The corresponding radar image is shown in Fig. 4. Each column of the image represents a single radar measurement. In total the image consists out of 466 measurements. The altitude of the radar measurement was extracted using a particle filter. For comparison the lidar data as well as the RTK GNSS altitude are plotted.

The stepped rock wall (step of $1.2 \mathrm{~m}$ ) was overflown the first time from $26 \mathrm{~s}$ until $28 \mathrm{~s}$, and the sharp edge (step of $0.4 \mathrm{~m}$ ) was overflown in the time slot from $28 \mathrm{~s}$ to $30 \mathrm{~s}$. As expected, the RTK GNSS system does not measure any altitude differences, since the altitude above mean sea level (MSL) stays almost unchanged.

The lag of the extracted radar data, in particular during the first overflight of the sharp edge, can be explained by the 


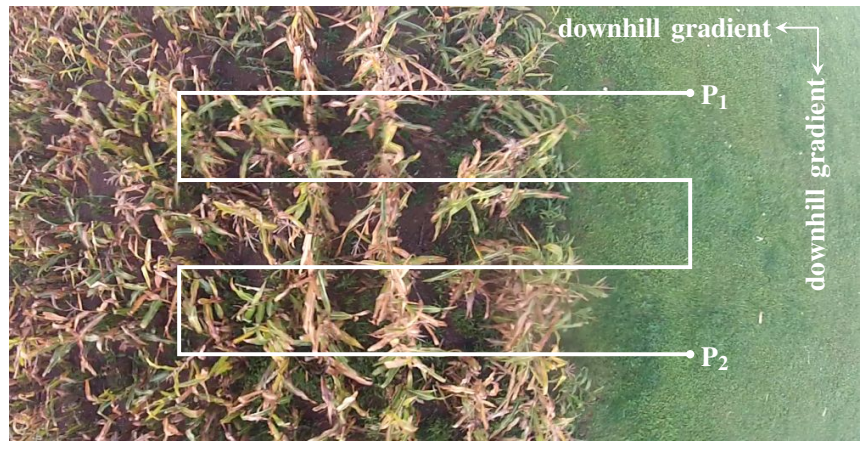

Fig. 5. Photo of the boundary between low grass and cornfield from the radar altimeters perspective. The flight path $\left(P_{1}\right.$ to $\left.P_{2}\right)$ as well as the slope gradient is illustrated.

inertia of the tracking algorithm and the large footprint of the radar beam. It can be seen that the stone slab at the edge of the rock wall leads to a strong reflection. As long as this strong reflection lies in the footprint of the antenna, the particle filter will track this reflection and not the weak reflection from the layer below. The same behaviour can be observed while flying over the stepped rock wall. The corners of the steps lead to a strong reflection delaying the radar signal. On the way back, the UAV speed was faster, so this effect is not visible.

The altitude above ground and the actual step size can be measured quite accurate. Due to the smaller footprint and the better resolution the performance of the lidar is advantageous in this scenario.

\section{EXPERIMENTAL RESUlTS - CORNFIELD}

In the second scenario the UAV was flown in a constant altitude above MSL in meanders at the boundary of a cornfield. The measuring field is illustrated in Fig. 5. Since the corn is sparsely planted, the ground is still visible in-between the corn rows.

The corresponding radar image is shown in Fig. 6. The sinusoidal curve of the absolute altitude can be explained by the slope gradient.

The radar image indicates, when the UAV is flying above the cornfield (e.g. between $12 \mathrm{~s}$ and $30 \mathrm{~s}$ ). The vegetation leads to reflection (clutter) in different distances closer than the ground reflection. Because of the large area illuminated by the radar and the ability to penetrate the corn leaves, the ground is visible in each measurement.

In Fig. 7 the particle filter extracted radar altitude and the raw lidar data are shown. Since the ground is visible in each radar measurement, the particle filter can track its reflection and determine the altitude above ground. However, the lidar with a small footprint only measures the distance between the $\mathrm{UAV}$ and the vegetation. It is not possible to determine the flight altitude above ground level. Thus, in this scenario the radar in conjunction with a particle filter can outperform the simple lidar sensor.

In Fig. 8 single radar measurements (envelope curves) with a temporal distance of $400 \mathrm{~ms}$ are shown. In the first measurement Fig. 8a the peak in a distance of $5.1 \mathrm{~m}$ represents

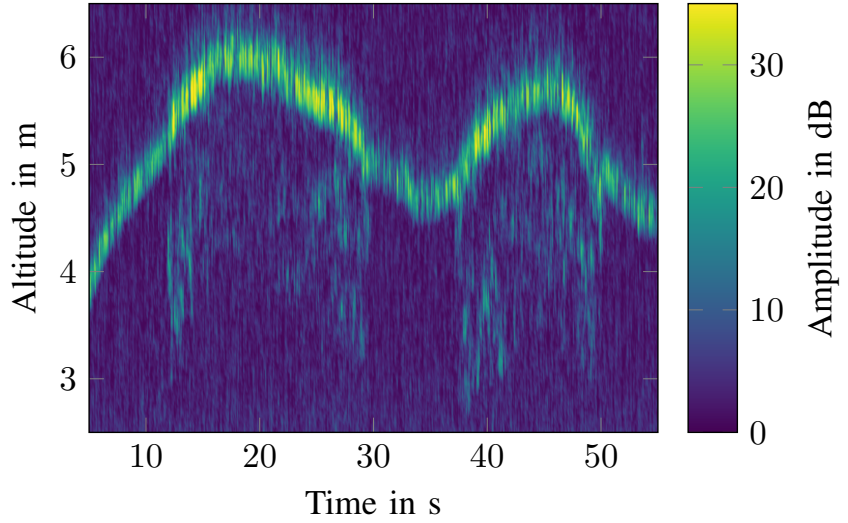

Fig. 6. Radar image from the flight above the cornfield.

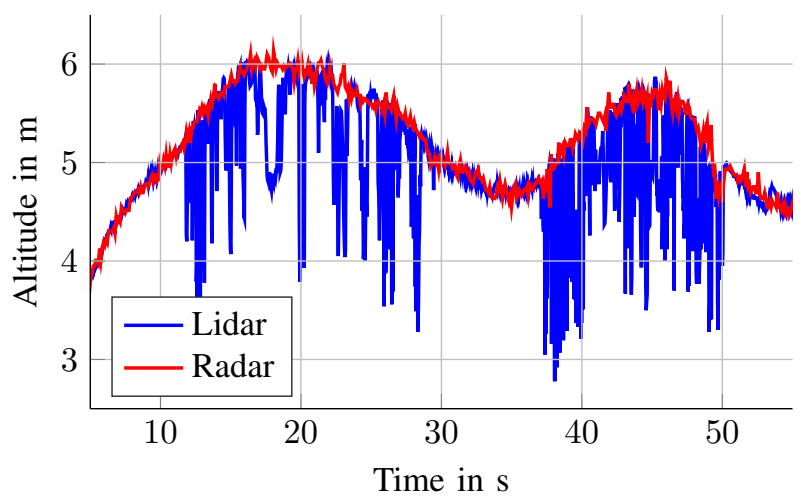

Fig. 7. Cornfield: comparison between lidar and radar.

the ground reflection. In the second measurement Fig. $8 \mathrm{~b}$ the altitude above ground is approximately $5.4 \mathrm{~m}$. The peaks in the range from $3 \mathrm{~m}$ to $4.5 \mathrm{~m}$ are reflections from vegetation, e.g. leaves, corncobs, and corn stalks. Regarding this, the closest reflection $3 \mathrm{~m}$ and the ground reflection $5.4 \mathrm{~m}$ the plant height could be estimated to $2.4 \mathrm{~m}$.
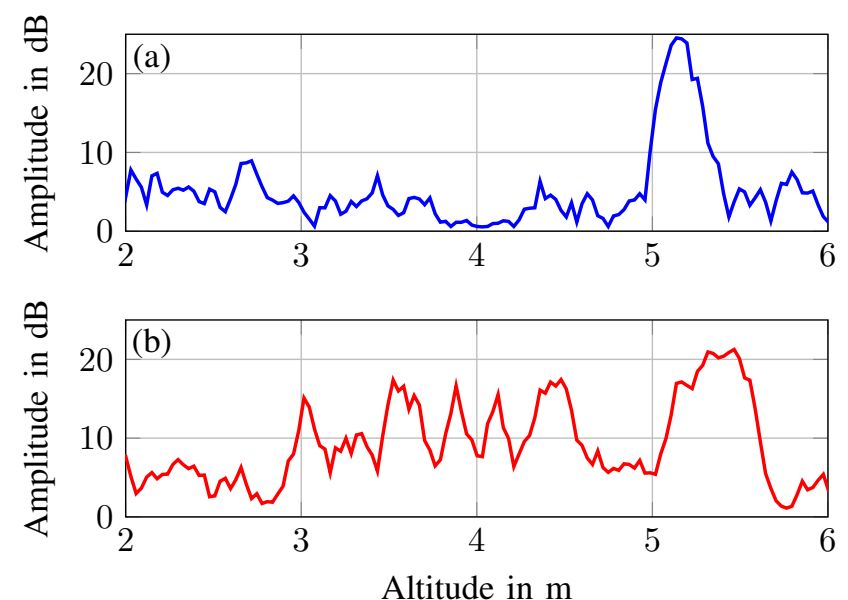

Fig. 8. Single radar measurements recorded in a temporal distance of $400 \mathrm{~ms}$ at the boundary of the cornfield. 


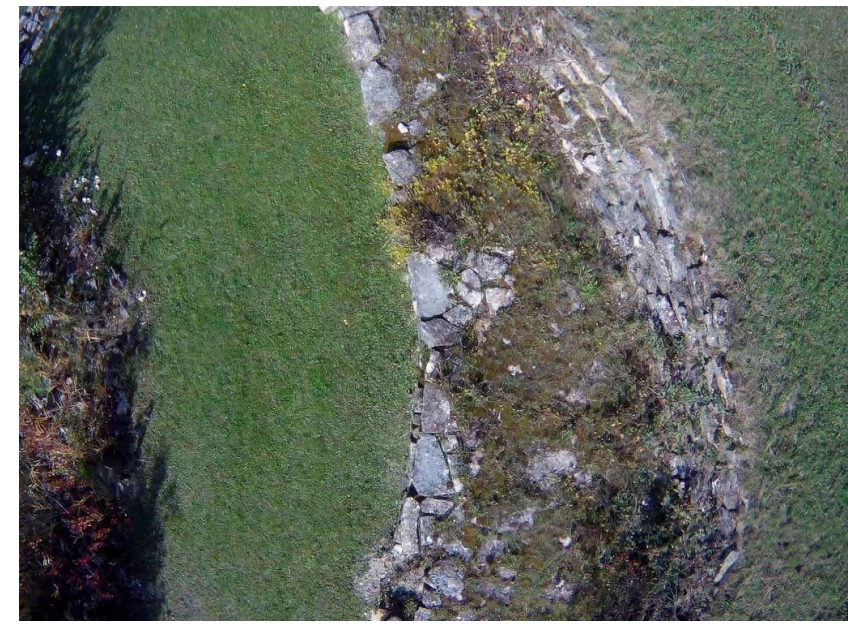

Fig. 9. Photo of the rock walls from the bird's eye view.

\section{TERRAIN MAPPING}

In many applications a 3D image is necessary, e.g. to estimate the volume of bulk solids, to create 3D building models, or to generate a digital elevation model (DEM). Using the system described above and performing a grid flight a 3D image can be generated. As example, a simple terrain map of the rock walls shown in Fig. 9 is presented.

To generate the image shown in Fig. 10, the UAV was manually steered several times above the rock walls in a constant altitude. The radar as well as the lidar provide only one altitude value per measurement point. Both sensors point downwards. They can not be steered into a certain direction to scan the area. Therefore, the resolution depends on the measurement grid, on the movement of the UAV and the beamwidth of the antenna.

The altitude above MSL obtained by the RTK GNSS was used as reference. Due to the different measuring rates, the position data was interpolated to fit to the radar measurements. As input data the raw lidar data and the altitude data of the radar sensor tracked with the particle filter were used.

In both terrain maps, the shape of the rock walls can be reproduced. As previously discussed, the particle filter tracks the strongest reflection. Thus, the edges of both rock walls smear. In addition, the reflection from the bush $(5.5 \mathrm{~m} / 8 \mathrm{~m})$, clearly visible in the lidar image, is not present in the radar image.

\section{CONCLUSION}

A low complexity radar sensor in conjunction with a particle filter tracking the UAV altitude above ground was presented. The comparison of the radar with a commercial low-cost lidar sensor in two different scenarios prove the functionality of the system. In sparse vegetation the radar sensor outperforms the lidar since the radar still can track the ground, whereas the lidar measures the distance to the vegetation.

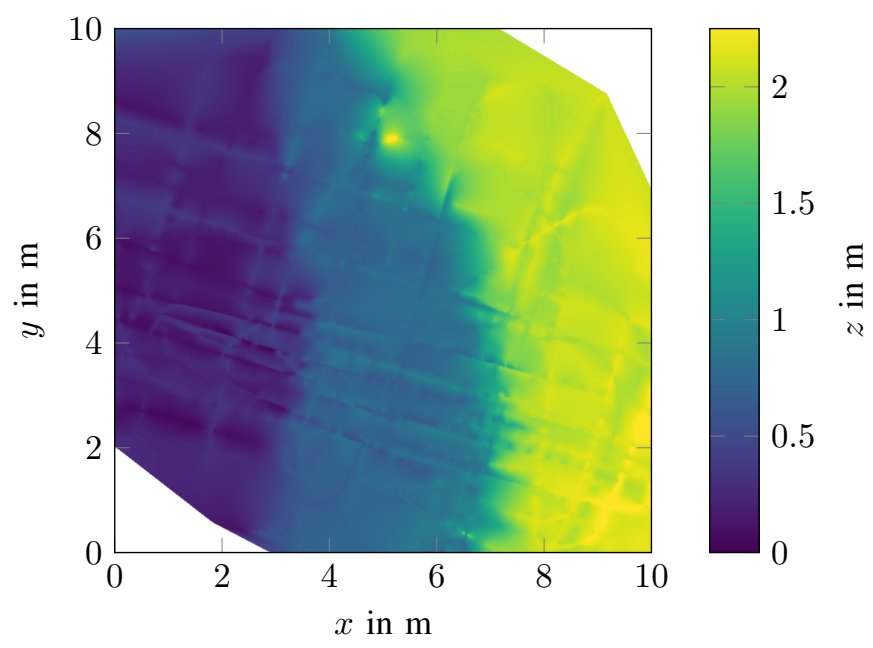

(a) Lidar.

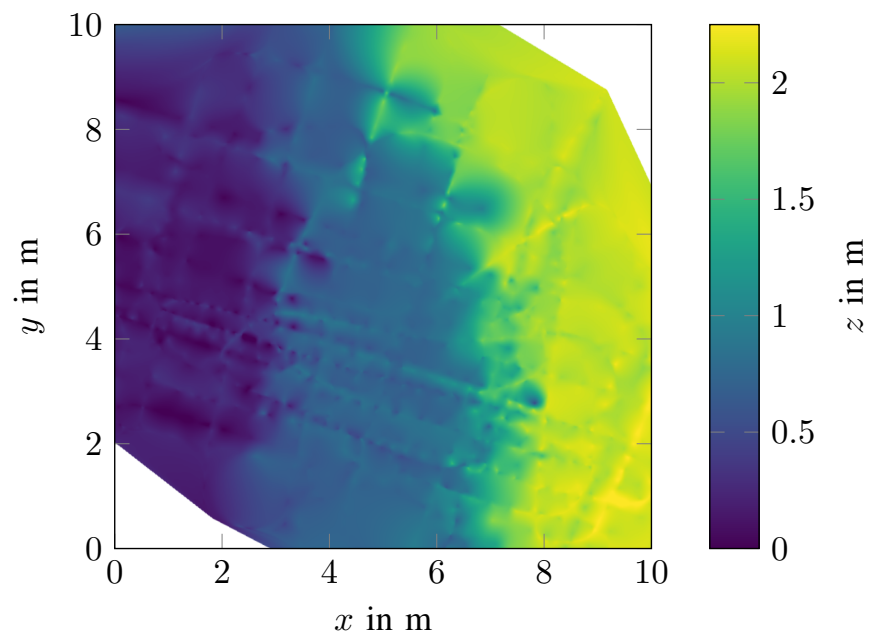

(b) Radar.

Fig. 10. Terrain maps of (a) lidar and (b) radar.

\section{ACKNOWLEDGEMENT}

The authors would like to thank the Urs Endress foundation for their support and the collaboration.

\section{REFERENCES}

[1] DJI, "Mavic Pro User Manual V1.6", http://www.dji.com/mavic/info 2017.

[2] Hügler P., Geiger M., and Waldschmidt C., "77 GHz Radar-Based Altimeter for Unmanned Aerial Vehicles" Radio \& Wireless Week $(R W W)$, California, 2018

[3] Schuetz M., Oesterlein M., Birkenhauer C., and Vossiek M., "A Custom Lightweight UAV for Radar Remote Sensing: Concept Design, Properties and Possible Applications", International Conference on Microwaves for Intelligent Mobility (ICMIM), Japan, 2017.

[4] Schartel M., Mayer W., and Waldschmidt C., "Digital True Time Delay for Pulse Correlation Radars," European Radar Conference (EuRAD), England, 2016.

[5] J. Motzer, "A pulse radar gauge for level measurement and process control", Microwave Symposium Digest. IEEE MTT-S International (Volume:3), 2000.

[6] Garmin, "Lidar Lite v3 Operational Manual and Technical Specification", 2016

[7] DJI, "D-RTK GNSS Quick Start Guide V1.0", https://www.dji.com/d-rtk/ info, 2016. 